\title{
An Efficient Spatial Temporal Provenance Mechanism for Adhoc Mobile Users
}

\author{
K Sai Divya, S.Siva Nageswara Rao, K. LakshmiNadh
}

\begin{abstract}
Location-based services rectangle measures quickly changing into vastly in different style. Additionally to services supported users' current location, several potential services believe users' currentlocation history, or their spatial-temporal place of origin.Malicious users might idle their spatial-temporal place of originwhile not a rigorously designed security system for users toprove their past locations. during this system, we tends to gift theSpatial-Temporal place of origin Assurance with Mutual Proofs theme. STAMP is meant for ad-hoc mobile usersgenerating location system proofs for every different in an exceedinglydistributed settings. However, it will simply accommodate trustyworthy mobile users and wireless access points. STAMP ensures theintegrity and non-transferability of the placement proofs andprotects users' privacy. A semi-trusted Certification Authority isemployed to distribute specific keys in addition as guard users against collusion by a light-weight entropy-based trust analysisapproach. Our image implementation is based on the Andriod platformshows that STAMP is low cost in terms of procedure and storageresources. Intensive simulation experiments show that ourentropy-based trust model is in a position to attains high collusion to detects the accuracy.
\end{abstract}

Keywords: Location proof, privacy, spatial-temporal provenance, trustworthy.

\section{INTRODUCTION}

As Location-Enabled mobile phones multiple area- $\mathrm{s}$ based on aministrations are quickly ending up noticeably tremendous mainstream. The greater part of the present area based on administrations for mobile phones depend on clients' at present area.Clients find their areas and import them to a server. Thus, the server performs calculation to view of the area data representations and returns the information or administrations to the clients. Not with standing clients' present areas, there is an expanded pattern and impetus to approved / not approved portable clients' past land areas. This opens a wide assortment of new area verification based on portableapplications. Saroiu et al. depicted a few such potentialapplications. Give us a chance let consider the three cases:

1. A store needs to offer rebates to regular clients. Clients must have the capacity to showconfirmation their hashed visits in the past to the store locations.

2. An organization which promotes the green driving and health may remunerate their representatives who walk or bicycle to work. The organization may energize every day strolling objectives of some settled number of miles.

Revised Manuscript Received on November 08, 2019.

K.Sai Divya Narasaraopeta Engineering College, Narasaraopet,522601, India.

Dr.S.Siva nageswararao, Associate Professor, Narasaraopeta Engineering College

Dr.K.LakshmiNadh, Associate Professor, Narasaraopeta Enginnering college
Workers need to demonstrate their past driving ways tothe organization alongside time history. This helps theorganization to reducing the healthcare, social insurance protection rates and move towards the practical way of lifestyle.

3. On the front line, when a scout gathering is conveyed toexecute a mission, the summoning focus may need eachfighter to keep a duplicate of their area follows for examination reason after the mission. The above applications oblige clients to have the capacity toget proofs from the areas they visit. Clients may then present atleast one of their verifications to an outsider verifier to asserttheir nearness at an area at a specific time. In this paper, wecharacterize the past areas of a versatile client at an arrangementof time focuses as the spatial-fleeting provenance (STP) of theclient, and an advanced confirmation of client's nearness at anarea at a specific time as STP evidence. Many works in literationhave alluded to such a proof as area verification. In this paper,we consider the two terms tradable. We inclinetowardSTPconfirmation" since it shows that such a proof is proposed forpast area visits with both spatial and fleeting data. Differentphrasings have been likewise utilized for comparative ideas, forexample, area guarantee, provenance verification and areajustification. Today's area construct benefits exclusively depends on light of clients' gadgets to decide their area, e.g., utilizing GPS communication. Be that as it may, it enables malevolent clients to fake theirSTP data. In this manner, we have to include outsiders in themaking of STP evidences keeping in mind the end goal toaccomplish the uprightness of the STP proofs. This, be that as itmay, opens various security and protection issues. To start with,including various gatherings in the system of STPconfirmationsmay imprenets clients' area security. Area data is very delicateindividual information. Knowing where a man was at a specifictime, one can gather his/her own exercises, politicalperspectives, wellbeing status, and dispatch spontaneouspublicizing, physical assaults or provocation. Subsequently,instruments to save clients' security and namelessness arerequired in a STP proof framework. Second, realness of STP evidences ought to be one of the primary outline objectives keeping in mind the end goal to accomplish honesty and non transferabilityof STP verifications. Also, it is conceivable thatvarious gatherings conspire and make fake STP proofs. Along these lines, cautious thought must be given to thecountermeasures against conspiracy assaults.

\section{RELATED WORK}

It introduces location proofs [1] a straight forward technique that allows the exposure of mobile apps that to securely prove their current locations and past locations. Author presents a concrete protocol which implements over $\mathrm{WiFi}$ in APs issue location proofs

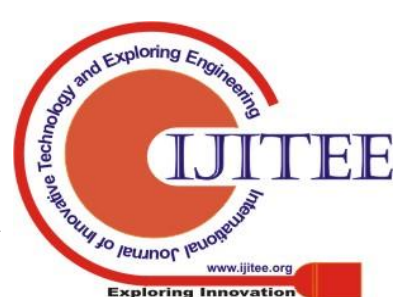


to mobile devices .A location proof is a piece of data that authorizes the location of a geographical area. Access points are (APs) embedding the location of a geographical area in location proofs, which are transmitted to designated recipient devices. A location proof has five fields: an issue, a recipient, a timestamp, a geographical location, and a digital signature. This system describes several potential applications where location proofs play a central role in enabling them like store discount so for loyal customers, green computing, reducing

fraud on auction websites, location-restricted content delivery and police investigations. This system has four security properties like integrity, non-transferability ,unforgeability, privacy.This system identified four challenges in designing alocation proof architecture and addressed them in VeriPlace[2].This system illustrated howcryptographic techniques can aid in preserving user privacy and protecting systemsecurity. VeriPlace system is a location proof architecturewhich is designed with protection of privacy and resilence of collision. This system requires three different trustedentities to provide security and privacy protection: a TTPL a UTTP ( User information managing the trusted third party)and a DAC ( Detection of cheating Authority). Every trustedpeople knows either a user's identity or his/her location, butnot both. VeriPlace's collusion detection works only if usersrequest their location proofs very frequently so that the longdistance between two location proofs that arechronologically close can be considered as anomalies. Thereare two benefits of this system like user privacy andcheating detection. Author discussed in detail about foursecurity challenges like privacy, security, flexibility, deployability.

\section{SYSTEM ARCHITECTURE}

Majority state art of mechanisms turned the attends to smallest wireless networks wireless LAN to cover indoor environments. They proposes a protocol that authorizes the mobile device to prove its presence to a Verifier with the help of an AP. The AP spots the Location Manager measures the round trip latency of request-reply protocol and based on time taken for the device to respond, it determines the location protocol. Echo protocol is also based on multiple transmitters and each of the transmitters must measure the round trip time with a specified precision. Our solution holds the existing Wi-Fi infrastructure, is not dependent or any additional entities to employ distance bounding protocol which requires significant changes to the hardware for proof generation. To build a secure location updating module first we utilize asymmetric encryption schemes. We introduce the encryption password based(EBP) method. It acts as the attribute that the user is set by the password and merges the random numbers (salt) for providing the data security. it does not have the theory of the confidential key because the extentof the confidential key affects the security of the method and it is difficult to remember.

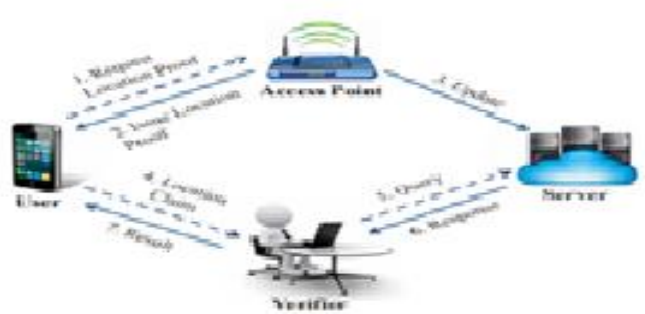

\section{Fig. 1: System Architecture \\ IV. PROPOSED SYSTEM}

Within the system, can you explains the beyond position to changes the past locations of a mobile user sequence of data distance points as the spatial-temporal source of a user, and a digital proof of user's presence at a location at a particular time as an STP proof. In this paper, we propose an STP proof scheme named Spatial-Temporal provenance Assurance with Mutual Proofs (STAMP). STAMP aims at ensuring the integrity and non-transferability of the STP proofs, with the capability of protecting users' privacy. We propose an entropy-based trust model to detect the collusion scenario. An issue of proof generations of STP protocol and verification protocol is introduced to achieve integrity and non transfer ability to generate STP proofs. it requires only single trusted third party which can embedded in Certificate Authority. STAMP is designed to maximize users' anonymity and location privacy. Users are given the control over the location granularity of their STP proofs. STAMP is collusion-resistant. At this moment doofus to impress the stamp resistant for collusion.distance bounding protocol is integrated into STAMP to prevents the proof collectors beyond the user. The eqivalent proposes a model for efficient trust models of based trust proposed to detect users mutually generating fake proofs for each other. STAMP uses a entropy based trust model to guard users from prover-witness collusion.This model also encourages witnesses against selfish behavior.

\subsection{Location Updating}

A locality position updates the system of a client responsible for preparing, scheduling, and sending location updates to the cloud system. It acts as data function of our tracking system. We provide several mechanisms to ensure three main objectives: (i) to inform the locality position should be sent to the cloud storage should be unidentified and unlikable (ii) future isolation and (iii)To investigate the reposition services to be own. To build a secure location updating module, first, we utilize asymmetric encryption schemes. We introduce the password-based encryption method. It has the following that user is set by the password and combines random numbers (salt) to provide the security of data. it does not have the concept of the secret key because the length of the secret key affects the security of the method and it is difficult to remember. The processes of encryption using PBE are as follows:

1.when the user is asked to set a password if ACT initlizes.

2After ACT collects a location coordinate, the location updating element to introduce the data encrypts with the help of a a salt and encrypts the data with the password.

3 masqurade sends the ciphertext for the network disk, and the salt will be appended to the location update.

4. After following the data packet has been sent, and locality and the salt will be deleted.

5.When the following locality position represents the 
another position . We can define Si stands for the state of a mobile device at the time $\mathrm{Ti}$, which contains several elements of ACT: P is the password of ACT, which is for encrypting the location. Li stands for location of the mobile device at the current time; $\mathrm{Ci}$ stands for the ciphertext, which contains location and time information encrypted Ri stands for the salt generation at the time $\mathrm{Ti}$, and it will be appended to $\mathrm{Ci}$ after it is used for encrypting.

\section{GLOBAL ATTENTION SCHEME}

In our model, each device allows describes the positions. It allows the system to register the devices like Bluetooth devices or WiFi access through MAC addresses and each one has an unique ID. A report of location from an X entity does not only contain its location, but also the MAC addresses sensed in the proximity. The report serves as a positive feedback for the trustworthiness of the entity if it complies with the ground truth. Similarly, it can be works as the negative feedback if it does not comply with the ground truth. When the positive computes the negative feedback is trivial when ground truth is available, in the setting, we do not have ground truth for the position of devices. Once a feedback graph is computed you can use exists the based on graph trust models to calculate the trustworthiness of the nodes in the graph. While various trust models are used in our system, there are two types of based on graph trust models and PeerTrust.

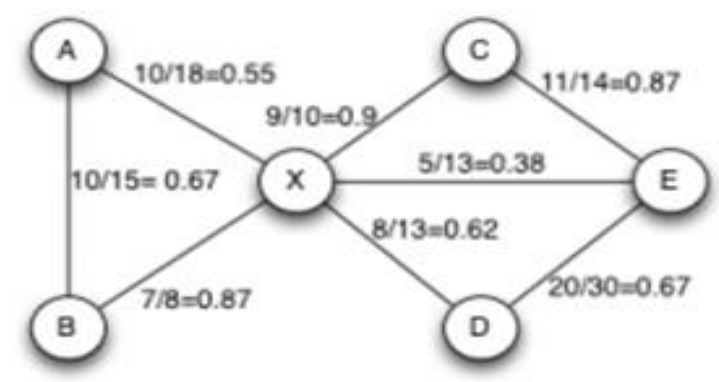

\section{METHODOLOGIES}

Fig. 2: Trust Feedback Graph.

Enabling Privacy-Preserving Location Proofs for Mobile Users Use the DES Algorithm. The user searched location is encrypted and that encrypted can be viewed only by the verifier and certificate authority, even the verifier and the certificate authority can view only in the form of encryption. Thus the location cannot be found by the third party or unknown person.

\section{EXPERIMENTAL EVALUATION}

Mobile device has limited resource when compared to ordinarycomputers through a lightweight client is consumption comprises of three components: consumption of power, storage system and the network traffic. It is not possible directly measure the consumption of power of ACT because it may not be accurate for some factors. To measure the battery overhead of ACT, webexperiments as follows: with and without ACT running on the mobile phone. We access the traffic of the network that ACT generates when using on a mobile device, which may connect to the Internet may connect to the Wireless networks or cellular network, and it taken care about the network traffic because it is related to the user.

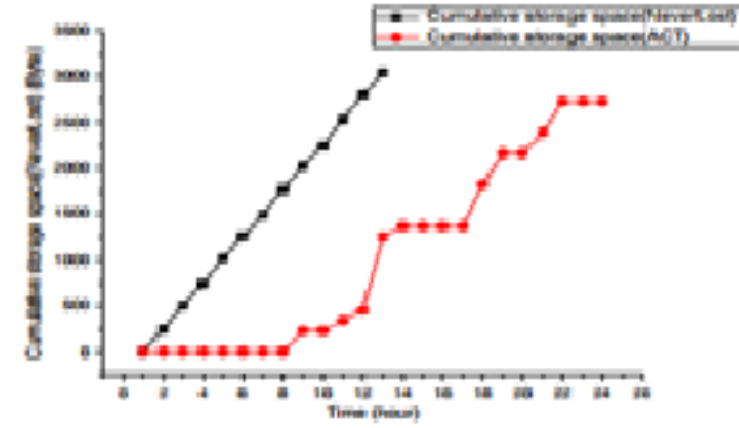

Figure 3. The storage space systemof Android CLOUD tracker running

\section{CONCLUSION AND FUTURE ENHANCEMENT}

In this proposed system a novel privacy-preserving location proof system based on a collaborative architecture. We can introduce the several techniques to attain both privacy and efficiency in this process and analyze their private properties. Using evaluation based on both synthetic and real-world LBSA traces, we also found that Location services provides a little computational and communication overhead to existing systems. In future we are going to increase the performance of access system that resolves the following issues indoor locating tamper apparent client efficient transfer line insight of locations etc.

\section{REFERENCES}

1. S. Saroiu and A. Wolman, "Enabling new mobile applications with location proofs," in Proc. ACM HotMobile, 2009, Art. no. 3.

2. W. Luo and U. Hengartner. Veriplace: a privacy-aware location proof architecture. In ACM GIS, 2010.

3. D. Singelee and B. Preneel, "Location verification using secure distance bounding protocols," in Proc. IEEE MASS, 2005.

4. B. Waters and E. Felten, "Secure, private proofs of location," Department of Computer Science, Princeton University, Princeton, NJ, USA, Tech. Rep., 2003

5. I. Afyouni, C. Ray, and C. Claramunt, "Spatial models for contextaware indoor navigation systems: A survey," J. Spatial Inf. Sci., no. 4, pp. 85-123, 2014.

6. N. Sastry, U. Shankar, and D. Wagner, "Secure verification of location claims," in Proc. ACM WiSe, 2003, pp. 1-10.

\section{AUTHORS PROFILE}

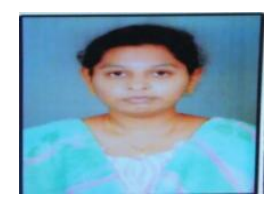

\begin{tabular}{llrr}
\multicolumn{1}{c}{ K.Sai } & Divya & ,pursuing & M.Tech in \\
computer science and engineering from & and \\
Narasaraopet & Engineering & college \\
,Narasaraopet,Guntur ,Andhra pradesh & 522601 \\
India.Her area of interest are web technology,oracle \\
and datamining.
\end{tabular}

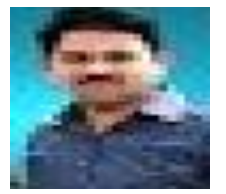

Dr. S. Siva Nageswara Rao qualified in Ph.D. in Computer Science \& Engineering from JNTUK, Kakinada. He received the B.Tech and M.Tech from JNTU, Hyderabad. $\mathrm{He}$ is at present working as Associate Professor in Narasaraopeta Engineering College. His research interests are Mobile Computing, Wireless Sensor Networks and Cloud Computing.

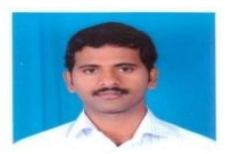

Dr.K.LakshmiNadh qualified in Ph.D. in Computer Science \& Engineering from JNTUK, Kakinada. He received the B.Tech and M.Tech from JNTU, Hyderabad. He is at present working as Associate Professor in Narasaraopeta Engineering College. His research

interests are Computer Networks, 
An Efficient Spatial Temporal Provenance Mechanism for Adhoc Mobile Users

Mobile Computing, Software Engineering, Agro Tech using IoT, Machine Learning and Deep Learning. He is a Coordinator for Entrepreneurship Development Centre and MOOCs Courses. 